Indian Journal of Science and Technology

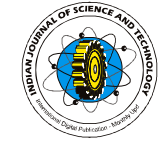

\title{
A low cost wavelet based mammogram image processing for early detection of breast cancer
}

\author{
D.M.Garge ${ }^{1}$ and V.N. Bapat ${ }^{2}$ \\ ${ }^{1}$ Deptt. of Electronics, Govt. Polytechnic, Kolhapur; ${ }^{2}$ A.D. College of Engineering, Ashta, India \\ vbkanhaji@gmail.com; dattagarge@yahoo.com
}

\begin{abstract}
We report a low cost wavelet based image processing technique using MATLAB for detecting the calcification on a relatively less sophisticated computing platform. The paper analyzed sample mammograms and reports the success of the algorithms as the unit cost of patient treatment is much lower than the existing one. In the existing scenario, there is a consistent increase in the unit cost of treatment to the patients owing to the requirement of the sophisticated computing hardware and Computer Aided Design Tools. Hence, the proposed low cost approach may benefit the marginalized sectors of the society.
\end{abstract}

Keywords: Mammograms, image processing, wavelet, MATLAB, cancer.

\section{Introduction}

Incidence of breast cancer in India is on the rise and is rapidly becoming the number one cancer in females pushing the cervical cancer to the second spot (Medindia, 2006). In India, the death toll due to the breast cancer is increasing at a rapid pace (Gajalakshmi et al., 2009). This warrants for early detection and diagnosis. Controlling the breast cancer has been a major challenge in India especially in case of marginalized women. Another factor making the scenario worst is the detection of the breast cancer at a very late stage. This is attributed to the lethargy of the Indian women towards the health care and regular check-up. The expensive health care system in India, especially in breast cancer treatment, probably puts enormous financial burden on the poor women. Simplified technology aid to the medical professionals especially in the rural areas may help the marginalized womenfolk. The present communication is in line with the traditional detection technique based on the mammogram, however with a difference in terms of the hardware and software required to formulate a complete healthcare setup for breast cancer detection.

Being an interdisciplinary

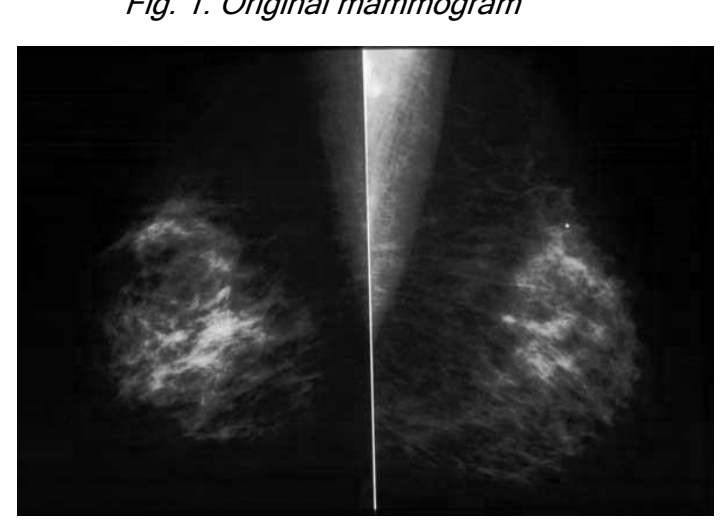

Fig. 2. Noisy mammogram

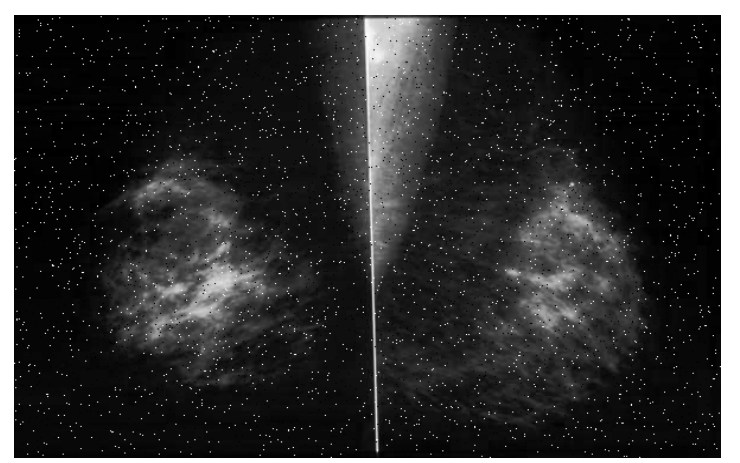

"Low-cost mammogram" http://www.indjst.org area of research, the mammograms and their analysis by the way of image processing, encompass many disciplines such as statistics, mathematics, computer and medical professionals. The role of application of statistical algorithm seems to be dominating. The pioneering work in this area is done by Chan et al. (1988) and Nishikawa et al. (1995) in applying the computer aided detection of the breast cancer. Later, the techniques are refined by using various methods such a heuristics, fuzzy reasoning, vector space machines etc. The research work seems to be forging on several directions such as conceiving improved algorithms, development of novel analytical framework, development of custom hardware based on programmable logic design and application of new tools such as Matlab and Simulink.

In spite of great deal of research work in this area (Sheshadri \& Kandaswamy, 2006; Jay Bhaskar \& Poonam Rani Gupta, 2009), there are still challenges lying ahead due to inherent limitations of the CAD tools in detecting the calcification patterns heterogeneous in several dimensions. As reported by Sentelle et al. (2002), size, shape and limited contrast from surrounding normal tissue, microcalcifications can occasionally be hard to detect in computer-aided detection (CAD) systems. Moreover, these CAD systems can also be slow compared to a radiologist's performance when reviewing film-screen mammography. The overall program size of the CAD tools compel the medical professionals to go for sophisticated computing system and thus lead to increase in the unit cost of the treatment.

The present communication reports the development of Mammogram Image Processing Algorithm using the tools such as Matlab and Simulink requiring less sophisticated computing hardware. The well established technique of mammogram image processing is used, however with the application of the improved algorithm and tools resulting in a better detection of the calcification.

\section{Methodology adopted}

The methodology comprises of the following sequence of steps: 
Fig. 3 Mammogram with wavelet filtering

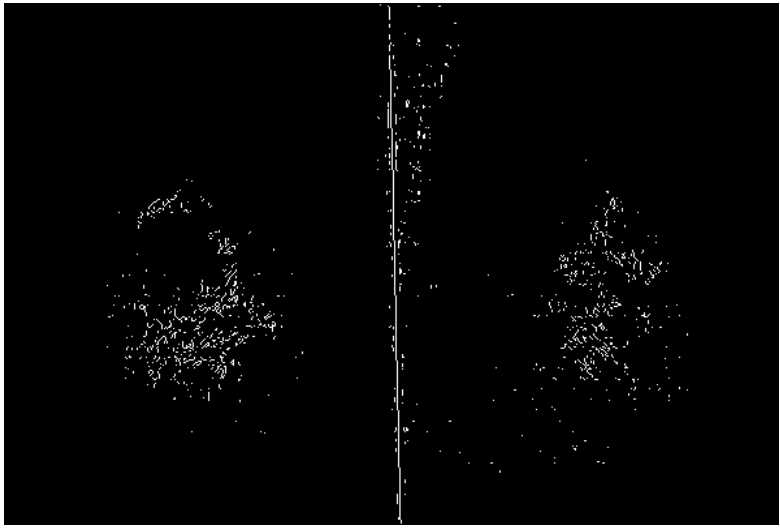

Fig.4. De-noised mammogram

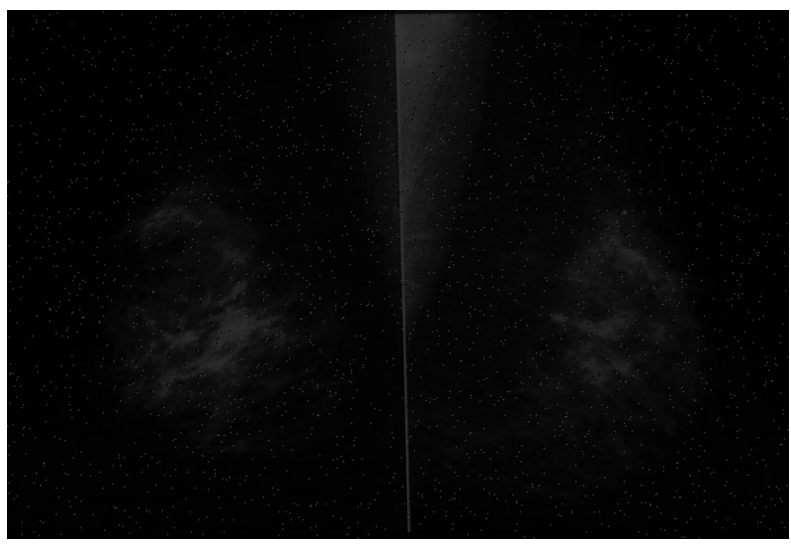

1.The test mammograms are taken from the database available at (www.cancer.org )

2. The test images mixed deliberately with the noise are then parsed through the High Pass and Low Pass filters implemented using the Matlab Version 6.5 to extract detailed coefficients and approximate coefficients respectively.

3. The denoised image is then reconstructed by using two methods. In the first only approximate coefficients were used while in other the threshold method is used.

4. The calcified portion of the mammogram was extracted by using the edge detection method and the same is presented on the monitor.

5. The mammogram is processed and it's horizontal and vertical gradients are used to extract horizontal and vertical features.

The setup required to run the algorithm mentioned under the methodology is less sophisticated and leads to cost effectiveness. The algorithm coded in the MATLAB Version 6.5 was executed successfully on the low cost PCs such as Zenith EcoStyle, HCL Neutron, HP Puppy model. The results are summarized in the following section.
Fig. 5. Mammogram with sobel filtering

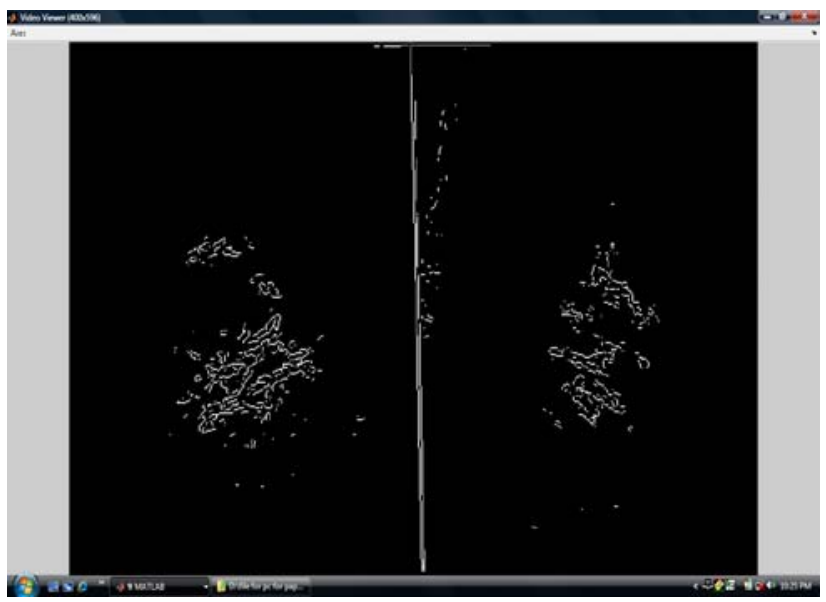

Fig.6. Edge detection of calcification

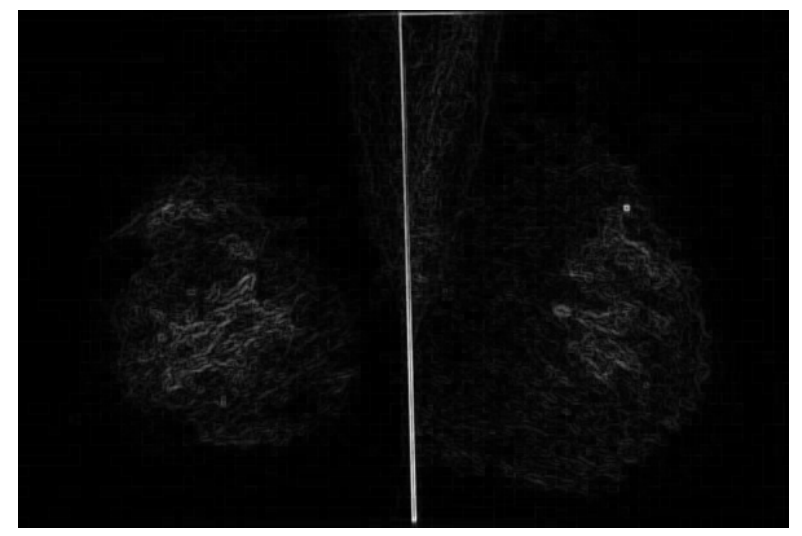

\section{Results and discussion}

The Fig.1-8 reveals the wavelet based processing of mammogram image obtained from web based database (www.cancer.org). Fig.1 reveals original mammogram showing micro calcification. This mammogram image is deliberately added with random noise of finite average value (Gaussian noise using Matlab in built function) as shown in Fig.2. Noisy mammogram is de-noised with threshold method has shown promising results (Fig.4). Using inbuilt function in MATLAB 'imfilter' with wavelet and sobel filter on mammogram image have shown interesting results as seen in Fig.3 and Fig. 5, where only calcified area from original image is extracted separately. Embedding the inbuilt functions in MATLAB, edges of calcified area in mammogram can be detected as depicted in Fig.6. Finally, the image matrix of mammogram is processed for the horizontal and vertical gradients to extract features as shown in Fig.7-8.

\section{Conclusion}

Sky rocketing cost of health care facilities especially for the breast cancer detection are prohibiting $(\sim 2000$ Rs.) the women patients to go for routine checkup. Moreover the rural health care units can't afford the expensive setup for detection of the breast cancer. This is
Research article

CIndian Society for Education and Environment (iSee)
"Low-cost mammogram" http://www.indjst.org
Garge \& Bapal Indian J.Sci.Technol. 
creating a sort of cost centric health care divide amongst the society. The present communication has successfully shown the possible cost reduction ( $\sim 60 \%$ cut) with the modification of algorithms without compromising the detection accuracy. Authors are working on further cost reduction by using the free open source software such as puppy Linux and Scilab that will make the facility more affordable for the masses.

Fig. 7. Mammogram with horizontal gradient

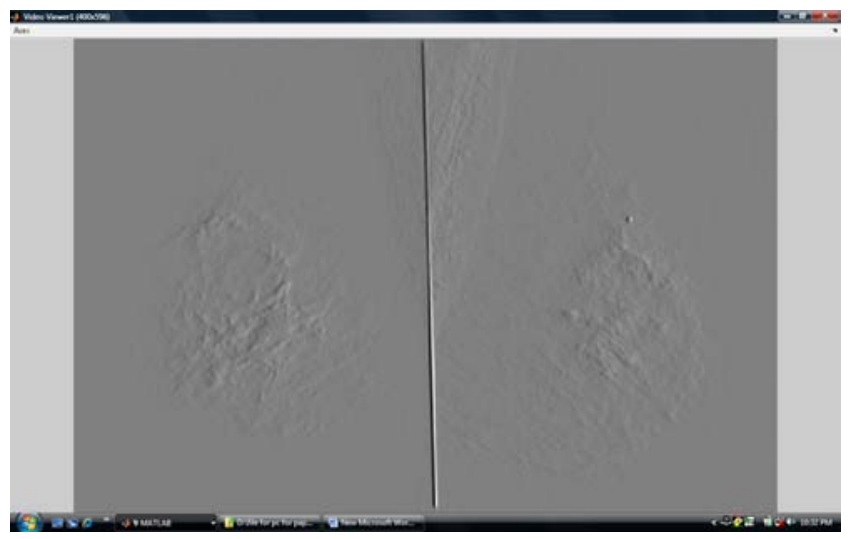

Fig.8. Mammogram with vertical gradient

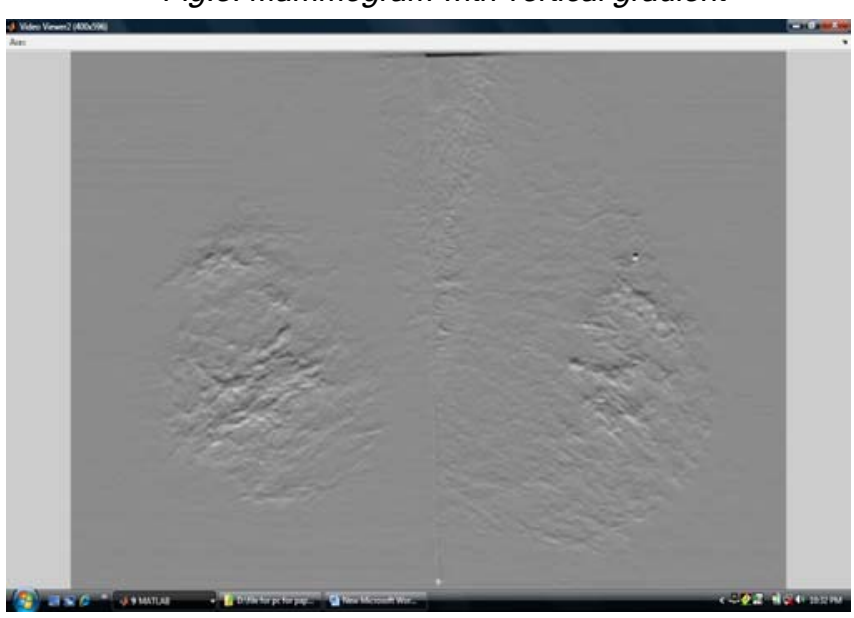

\section{References}

1. Chan HP, Doi K, Vyborny CJ, Lam KL and Schmidt RA (1988) Computer-aided detection of microcalcications in mammograms: methodology and preliminary clinical study. Investigative Radiol. 23, 664-671.

2. Gajalakshmi Vendhan, Aleyamma Mathew, Paul Brennan, Balakrishnan Rajan, Vendhan C. Kanimozhi, Anitha Mathews, Beela S. Mathew and Paolo Boffetta (2009) Breastfeeding and breast cancer risk in India: a multicenter case-control study. Intl.J.Cancer. 125 (3), 662-665.

3. Jay Bhaskar and Poonam Rani Gupta (2009) Design of an automated cancer detection system. Proc. ASCNT-2009, CDAC, Noida, India. pp: 9- 5.
4. Medindia (2006) Breast cancer in India rising rapidly. http://www.medindia.net/news/view_news_main. Jan 22.

5. Nishikawa RM, Jiang $Y$, Ginger ML, Schmidt RA, Vyborny CJ and Zhang W (1994) Performance of automated CAD schemes for the detection and classification of clustered microcalcifications. In: Proc. $2^{\text {nd }}$ Intl. Workshop on Digital Mammography, York, England. pp: 13-20.

6. Sentelle S, Sentelle C and Sutton MA (2002) Multiresolution-based segmentation of calcifications for the early detection of breast cancer. Real Time Imaging. 8 (3), 237-252.

7. Sheshadri HS and Kandaswamy A (2006) Computer aided decision system for early detection of breast cancer. Indian J. Med. Res. 124, 149-154. 